\title{
Attenuation of Adenosine-Induced Myocardial Perfusion Heterogeneity by Atenolol and Other Cardioselective $\beta$-Adrenoceptor Blockers: A Crossover Myocardial Perfusion Imaging Study
}

\author{
Eliana Reyes ${ }^{1,2}$, James Stirrup ${ }^{1,2}$, Michael Roughton $^{3}$, Savio D’Souza ${ }^{4}$, S. Richard Underwood ${ }^{1,2}$, and \\ Constantinos Anagnostopoulos 2,5 \\ ${ }^{I}$ National Heart and Lung Institute, Imperial College London, London, United Kingdom; ${ }^{2}$ Nuclear Medicine Department, Royal \\ Brompton Hospital, London, United Kingdom; ${ }^{3}$ Research and Development Office, Royal Brompton Hospital, London, United \\ Kingdom; ${ }^{4}$ Department of Cardiology, Royal Brompton Hospital, London, United Kingdom; and ${ }^{5}$ Barts and the London School of \\ Medicine and Dentistry, St. Bartholomew's Hospital, London, United Kingdom
}

\begin{abstract}
Little is known about the effect of chronic $\beta$-blockade on adenosine actions. We sought to investigate the effect of oral $\beta$-blockers on the presence, extent, and severity of myocardial perfusion abnormality induced by adenosine in patients with coronary artery disease. Methods: In this crossover study, 45 male patients with coronary artery disease on $\beta$-blocker therapy with atenolol, bisoprolol, or metoprolol underwent adenosine myocardial perfusion imaging both on and off $\beta$-blockade in a random order on separate days. Myocardial perfusion was assessed both qualitatively and quantitatively. Hemodynamic response, image analysis, and sensitivity for the detection of coronary stenosis $(\geq 50 \%$ luminal diameter reduction on $x$-ray coronary angiography) were compared between the on and off $\beta$-blocker studies. Results: Rate pressure product both at baseline and at peak adenosine infusion decreased by $23 \% \pm$ $15 \%$ and $21 \% \pm 18 \%$, respectively, after $\beta$-blockade $(P<$ 0.001 for all). The median (interquartile range) summed difference score, a measure of defect reversibility, and quantitative defect size were both significantly lower after $\beta$-blockade (median, 7.0 [interquartile range, 2.0-9.5] vs. median, 5.0 [interquartile range, $0-8.0$ ], $P=0.002$; and quantitative defect size, $18 \%$ [interquartile range, $9 \%-34 \%$ ] vs. quantitative defect size, $6 \%$ [interquartile range, $0 \%-19 \%$ ], $P<0.001$, respectively). The overall sensitivity for the detection of coronary stenosis decreased from 0.76 (95\% confidence interval, $0.65-0.88$ ) to 0.58 (95\% confidence interval, 0.45-0.71) after $\beta$-blockade $(P=0.03)$. Conclusion: $\beta$-blockade causes a small but significant reduction in the extent and severity of perfusion abnormality by adenosine. This may reduce the diagnostic sensitivity of adenosine myocardial perfusion imaging for the detection of flow-limiting coronary stenosis.
\end{abstract}

Received Dec. 16, 2009; revision accepted Mar. 16, 2010. For correspondence or reprints contact: Eliana Reyes, Department of Nuclear Medicine, Royal Brompton Hospital, Sydney St., London SW3, 6NP U.K.

E-mail: e.reyes@rbht.nhs.uk

COPYRIGHT @ 2010 by the Society of Nuclear Medicine, Inc.
Key Words: $\beta$-blocker; adenosine; coronary artery disease; myocardial perfusion imaging

J Nucl Med 2010; 51:1036-1043

DOI: 10.2967/jnumed.109.073411

$\mathbf{I}_{\mathrm{n}}$ the management of angina, $\beta$-adrenoceptor blockers are highly effective, but their use may affect the diagnostic accuracy of tests intended to detect the functional consequences of coronary artery disease (CAD). By preventing an increase in myocardial oxygen demand, $\beta$-blockers attenuate exercise-induced ischemia (1), and this attenuation may result in underestimation of the presence of disease by exercise testing. An advantage of myocardial perfusion imaging (MPI) is that it can be combined with pharmacologic stress agents in patients in whom dynamic exercise may be inadequate $(2,3)$. Until recently, it was thought that $\beta$-blockers had no effect on the action of primary coronary vasodilators (1). However, studies with dipyridamole have challenged this view $(4,5)$. Treatment with a $\beta_{1}$-adrenoceptor blocker attenuated the coronary hyperemic response to dipyridamole as measured by ${ }^{13} \mathrm{~N}$-ammonia PET $(6)$ and reduced the magnitude of inducible perfusion abnormalities on dipyridamole MPI (4). Although evidence from the dipyridamole studies strongly suggests that $\beta$-blockade may also affect the response to adenosine, recent observations do not support this $(7,8)$.

There are no prospective data about the effect of $\beta$-blockers on adenosine MPI. Until such data become available, discontinuation of $\beta$-blockers before adenosine vasodilation cannot be justified. The aim of this study was to test the hypothesis that $\beta$-blockade would reduce the 
extent and severity of inducible perfusion abnormality by adenosine and that this would decrease the sensitivity of adenosine MPI for the detection of hemodynamically significant coronary artery stenosis. The proof of our hypothesis was achieved by comparing the size and depth of adenosine-induced abnormalities in the same subject before and after discontinuation of $\beta$-blocker medication.

\section{MATERIALS AND METHODS}

\section{Patient Population}

Patients were recruited from those referred to the Royal Brompton Hospital for pharmacologic MPI with adenosine. Patients with clinically suspected myocardial ischemia and documented CAD were enrolled if they were on a $\beta_{1}$-selective adrenoceptor blocker for the management of anginal symptoms. Patients were excluded from the study if they had a documented history of acute myocardial infarction, unstable angina, or coronary revascularization within $8 \mathrm{wk}$ before enrollment; contraindication to adenosine stress (i.e., bronchospasm, secondor third-degree atrioventricular block without a functioning pacemaker, sick sinus syndrome); or any condition in which discontinuation of $\beta$-blockade therapy was contraindicated (i.e., tachyarrhythmias, ongoing Canadian Cardiovascular Society classification grades III-IV angina (9), uncontrolled or poorly controlled hypertension). The second MPI procedure was performed after a washout or treatment period of a minimum of $5 \mathrm{~d}$ but no longer than $6 \mathrm{wk}$. Coronary anatomy data were available in a subset of patients who underwent $\mathrm{x}$-ray coronary angiography within 6 mo before or after the study.

Fifty patients were enrolled in the study. Both male and female patients were initially recruited, but only men completed both MPI studies. The Royal Brompton and Harefield Research Ethics Committee approved the study, and written informed consent was obtained from all patients.

\section{Study Design}

In this randomized, crossover study, eligible patients underwent 2 identical adenosine MPI studies-1 after discontinuation of $\beta$-blocker and 1 during $\beta$-blocker medication-in random order on separate days. The study was not masked to the patient or the investigator assigning treatment and conducting the adenosine MPI study but was masked to the investigators interpreting the images.

\section{Adenosine Stress}

For each study, patients abstained from caffeine-containing products for at least $12 \mathrm{~h}$ before adenosine stress. Patients were instructed to stop calcium antagonists for $24 \mathrm{~h}$, and nitrate preparations were withheld the morning of the test. For the off- $\beta$-blocker study, $\beta$-blockade medication was stopped before the stress test for an interval equivalent to 5 half-lives. For the on- $\beta$-blocker study, patients were instructed to continue their $\beta$-blocker medication as prescribed by their physician. For both studies, adenosine was administered intravenously at $140 \mu \mathrm{g} / \mathrm{kg} /$ $\min$ for 6 min combined with low-level exercise on a bicycle ergometer.

The electrocardiogram (ECG) rhythm strip was monitored throughout each stress procedure, and blood pressure and heart rate were recorded every $2 \mathrm{~min}$ until completion of the test. An ischemic ECG was defined by the presence of horizontal or downsloping ST-segment depression greater than $1 \mathrm{~mm}$ at $80 \mathrm{~ms}$ after the $\mathrm{J}$ point for at least 3 consecutive beats in at least 1 lead. Symptoms were recorded as reported by the patient, who was also questioned directly about symptoms every 2 min until completion of the test. Patient symptoms were graded at the time of testing using a symptom-severity score from 0 (none) to 3 (severe). A summed score was obtained by adding the score for the commonest symptoms (incidence $\geq 5 \%$ ). All events during or after the stress test that warranted medical intervention were recorded as adverse events. Safety was assessed by the collection of vital signs, ECG data, and adverse events during each stress procedure and by a telephone interview conducted $24-48 \mathrm{~h}$ after each MPI procedure.

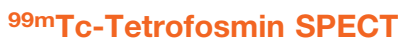

The same image acquisition parameters and processing were used for both MPI studies. For the stress images, 250-350 $\mathrm{MBq}(6.8-9.4 \mathrm{mCi})$ of ${ }^{99 \mathrm{~m}} \mathrm{Tc}$-tetrofosmin were injected $3 \mathrm{~min}$ into the adenosine infusion, and image acquisition started $45 \mathrm{~min}$ later. For the rest images, $750-1,000 \mathrm{MBq}(20.3-27 \mathrm{mCi})$ of ${ }^{99 \mathrm{~m}} \mathrm{Tc}$-tetrofosmin were injected $2-3 \mathrm{~h}$ after the stress injection preceded by $800 \mu \mathrm{g}$ of sublingual glyceryl trinitrate, and imaging was performed 45 min later with ECG gating using 16 frames per cardiac cycle. To minimize radiation exposure to the patient, rest imaging was not repeated. SPECT was performed using a dual-head $\gamma$-camera (Ventri; GE Healthcare) equipped with a high-resolution collimator for ${ }^{99 \mathrm{~m}} \mathrm{Tc}$ imaging. Transverse tomograms of the left ventricle were reconstructed using a Butterworth filter with a cutoff frequency of 0.5 cycles $/ \mathrm{cm}$, order of 5 , during iterative reconstruction (Hermes Medical Solutions), and transaxial slices were reoriented to obtain short-axis, horizontal long-axis, and vertical long-axis views of the left ventricle according to current recommendations (10). Unprocessed planar images were reviewed to exclude significant patient motion, softtissue attenuation, or diaphragmatic attenuation.

The images were interpreted independently by 2 experienced nuclear cardiologists without knowledge of $\beta$-blockade status or clinical or angiographic data. Disagreement was resolved by consensus. Tomograms were divided into 17 segments according to the American Heart Association model (10) and tracer uptake graded semiquantitatively for each segment. Scores were attributed on a 5-point system by taking into account the severity of the perfusion defect: 0 , normal uptake (tracer activity $\geq 70 \%$ of maximal myocardial activity); 1 , mild reduction $(50 \%-69 \%) ; 2$, moderate reduction $(30 \%-49 \%)$; 3 , severe reduction $(10 \%-29 \%)$; and 4 , absence of uptake (tracer activity $0 \%-9 \%$ of maximum). Account was taken of artifacts such that a segment with normal uptake but reduced counts by attenuation was scored as 0 . Hence, total stress and rest scores varied from 0 to a theoretic maximum of 68. For each patient, the summed stress score (SSS) and summed rest score were obtained by adding the perfusion score for each segment. The summed difference score (SDS) was obtained by subtracting the SSS from the summed rest score, and this measure was used to define the size and depth of reversibility. A score was also assigned for overall image quality: 0 (inadequate), 1 (poor), 2 (adequate), and 3 (good). Motion correction and visual and quantitative (Quantitative Gated SPECT; Cedars-Sinai Medical Center) assessment of contractile function aided the interpretation of the perfusion findings.

Quantitative defect size was calculated using a commercially available program (Quantitative Perfusion SPECT; Cedars-Sinai 


\section{TABLE 1. Baseline Characteristics ( $n=45 \mathrm{Men})$}

\begin{tabular}{|c|c|c|}
\hline Demographic & $n$ & $\%$ \\
\hline \multicolumn{3}{|l|}{ Cardiac risk factors } \\
\hline Hypertension & 19 & 42 \\
\hline Hypercholesterolemia & 28 & 62 \\
\hline Smoking & 10 & 22 \\
\hline Diabetes mellitus & 12 & 27 \\
\hline Prior myocardial infarction & 5 & 11 \\
\hline Prior percutaneous coronary intervention & 4 & 9 \\
\hline Prior coronary artery bypass grafting & 7 & 16 \\
\hline \multicolumn{3}{|l|}{$\beta$-blocker } \\
\hline Atenolol & 32 & 71 \\
\hline Bisoprolol & 10 & 22 \\
\hline Metoprolol & 3 & 7 \\
\hline \multicolumn{3}{|l|}{ Other medication } \\
\hline Calcium antagonist & 11 & 24 \\
\hline Nitrates & 18 & 40 \\
\hline Nicorandil & 12 & 27 \\
\hline Angiotensin-converting enzyme inhibitor & 27 & 60 \\
\hline Aspirin & 38 & 84 \\
\hline Statin & 42 & 93 \\
\hline $\begin{array}{l}\text { Values are mean } \pm \text { SD. Mean age of men } \\
65 \pm 9 \text {, and mean body mass index }\left(\mathrm{kg} / \mathrm{m}^{2}\right)\end{array}$ & udy & $\begin{array}{l}\text { was } \\
\pm 5\end{array}$ \\
\hline
\end{tabular}

Medical Center) and expressed as a percentage of the myocardium involved in 1 of the 3 main coronary vascular territories: left anterior descending, left circumflex, or right coronary artery territory. The extent of reversibility was calculated by subtracting the extent of defect on the stress images from the extent of defect on the resting scan.

\section{X-Ray Coronary Angiography}

If clinically indicated, coronary angiography was performed using the standard Judkins technique, and the images were interpreted qualitatively by an experienced reader who had no knowledge of patient clinical status or scintigraphic results.
Coronary angiograms were included for analysis if they were performed within 6 mo before or after the MPI study, and no coronary intervention was undertaken between the angiographic and scintigraphic procedures. For the purpose of the study, an angiographically significant coronary stenosis was defined as greater than or equal to $50 \%$ luminal diameter narrowing of a major epicardial coronary artery. Results were used to estimate overall and regional (individual vessel) sensitivity.

\section{Statistical Analysis}

Data were summarized as mean value \pm SD of the mean if normally distributed and as median (range or interquartile range) if not. The primary outcome measure of the study was the extent and severity of reversible perfusion abnormality (as SDS) on adenosine MPI images. Secondary outcome measures were the sensitivity of adenosine MPI for the detection of individual coronary stenosis in the presence and absence of $\beta$-blockade therapy, hemodynamic response, and electrocardiographic changes during adenosine in the presence and absence of $\beta$-blocker. Hemodynamic and scintigraphic variables were compared using linear mixed-model analysis. Post hoc comparisons were performed using either a paired $t$ test or the Wilcoxon signed-rank test if the data were nonnormally distributed. Categoric variables were compared using the McNemar test. Differences were considered statistically significant at $P$ values less than 0.05 (2-sided). Analysis was performed using SPSS 14.0 (SPSS Inc.).

An SDS difference of 2 or greater was considered significant. After correction for nonnormality based on Pitman's asymptotic relative efficiency, 45 patients were needed for a probability of $90 \%$ of detecting such a difference at a 2 -sided $5 \%$ significance level. Considering a dropout rate of approximately $15 \%$, a total of 52 patients were estimated as needed to enter the study.

\section{RESULTS}

\section{Patient Characteristics}

Patient characteristics at baseline are summarized in Table 1. Of the initial 50 patients enrolled, 45 patients

\section{TABLE 2. Hemodynamic Response to Adenosine Before and After $\beta$-Blockade}

\begin{tabular}{|c|c|c|c|}
\hline Test & Off $\beta$-blocker & On $\beta$-blocker & $P$ \\
\hline \multicolumn{4}{|l|}{ Rest } \\
\hline Heart rate (bpm) & $73 \pm 11$ & $57 \pm 5$ & $<0.001$ \\
\hline Systolic blood pressure $(\mathrm{mm} \mathrm{Hg})$ & $139 \pm 21$ & $136 \pm 20$ & 0.3 \\
\hline Diastolic blood pressure $(\mathrm{mm} \mathrm{Hg})$ & $84 \pm 11$ & $83 \pm 12$ & 0.8 \\
\hline Rate-pressure product $\left(\times 10^{3} \mathrm{~mm} \mathrm{Hg} \cdot \mathrm{min}^{-1}\right)$ & $10.1 \pm 2.3$ & $7.7 \pm 1.3$ & $<0.001$ \\
\hline \multicolumn{4}{|l|}{ Peak stress } \\
\hline Heart rate $(\mathrm{bpm})$ & $97 \pm 14^{*}$ & $81 \pm 9^{\star}$ & $<0.001$ \\
\hline Systolic blood pressure (mm Hg) & $147 \pm 27^{*}$ & $140 \pm 24$ & 0.04 \\
\hline Diastolic blood pressure $(\mathrm{mm} \mathrm{Hg})$ & $83 \pm 11$ & $80 \pm 13$ & 0.3 \\
\hline Rate-pressure product $\left(\times 10^{3} \mathrm{~mm} \mathrm{Hg} \cdot \mathrm{min}^{-1}\right)$ & $14.3 \pm 3.2^{*}$ & $11.3 \pm 2.4^{*}$ & $<0.001$ \\
\hline Workload (watts) ${ }^{\dagger}$ & $5(0-8.8)$ & $5(0-10)$ & 0.2 \\
\hline$\Delta$ Heart rate $(\mathrm{bpm})$ & $24 \pm 11$ & $24 \pm 8$ & 0.6 \\
\hline$\Delta$ Systolic blood pressure $(\mathrm{mm} \mathrm{Hg})$ & $9 \pm 16$ & $5 \pm 15$ & 0.2 \\
\hline$\Delta$ Diastolic blood pressure $(\mathrm{mm} \mathrm{Hg})$ & $-1 \pm 10$ & $-3 \pm 12$ & 0.5 \\
\hline$\Delta$ Rate-pressure product $\left(\times 10^{3} \mathrm{~mm} \mathrm{Hg} \cdot \mathrm{min}^{-1}\right)$ & $4.2 \pm 2.2$ & $3.6 \pm 1.9$ & 0.07 \\
\hline
\end{tabular}

${ }^{\star} P \leq 0.001$ vs. rest values.

${ }^{\dagger}$ Workload data summarized as median, with interquartile ranges in parentheses.

Exercise was not indicated in 1 patient because of left bundle branch block. bpm = beats per minute. 


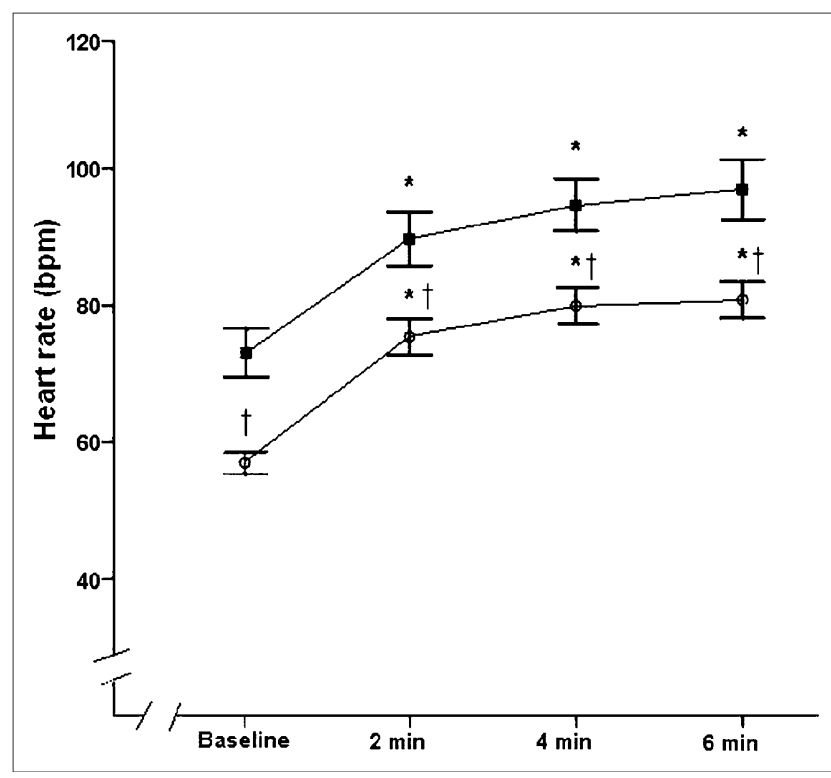

FIGURE 1. Mean heart rate at baseline and during adenosine stress before $(\square)$ and after $(\bigcirc) \beta$-blockade. Error bars represent $95 \%$ confidence interval. ${ }^{\star} P<0.001$ vs. baseline. ${ }^{\dagger} P<0.001$ vs. off $\beta$-blockers. bpm $=$ beats per minute.

(mean age $\pm \mathrm{SD}, 65 \pm 9 \mathrm{y}$ ) completed both adenosine MPI procedures at a median interval of $13 \mathrm{~d}(5-31 \mathrm{~d})$. Atenolol was taken by most patients $(n=32,71 \%)$, followed by bisoprolol $(n=10,22 \%)$ and metoprolol $(n=3,7 \%)$. The median daily dose was $50 \mathrm{mg}$ (range, $25-100 \mathrm{mg}$ ) for atenolol, $2.5 \mathrm{mg}$ (range, $1.25-10 \mathrm{mg}$ ) for bisoprolol, and 25 $\mathrm{mg}$ (range, 12.5-25 mg) for metoprolol. Almost half the patients $(42 \%)$ were under consideration for coronary revascularization at the time of the study.

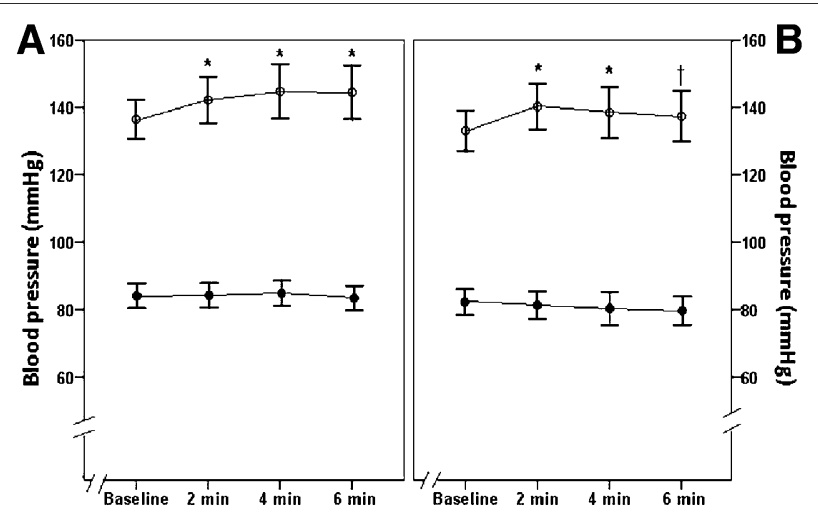

FIGURE 2. Mean systolic ( $\bigcirc$ ) and diastolic (O) blood pressure at baseline and during adenosine stress off $\beta$-blockers $(A)$ and on $\beta$-blockers (B). Error bars represent $95 \%$ confidence interval. ${ }^{\star} P<0.02$ vs. baseline. ${ }^{\dagger} P=0.04$ vs. off $\beta$-blockers.

\section{Hemodynamic Response}

The resting heart rate decreased significantly from $73 \pm$ 11 beats per minute off $\beta$-blockers to $57 \pm 5$ beats per minute on $\beta$-blockers $(P<0.001)$. $\beta$-blockade also decreased heart rate during adenosine stress $(P<0.001$; Table 2; Fig. 1), but the magnitude of change from rest to peak stress was not significantly different between the offand on- $\beta$-blocker status (Table 2 ). The systolic blood pressure response to adenosine also decreased with $\beta$-blockade $(P=0.005$; Fig. 2$)$. Rate-pressure product both at baseline and at peak adenosine infusion decreased by $23 \% \pm 15 \%$ and $21 \% \pm 18 \%$, respectively, after $\beta$-blockade $(P<0.001$ for all; Table 2$)$.

Extent and Severity of Myocardial Perfusion Abnormality

The extent and severity of adenosine-induced perfusion abnormality decreased after $\beta$-blockade (median SSS, 12.0 [interquartile range, 7.0-17.0] vs. 9.0 [interquartile range, 6.0-17.5]; $P=0.03$; Table 3). Defect reversibility also decreased with $\beta$-blockade (SDS, 7.0 [interquartile range, $2.0-9.5$ ] vs. 5.0 [interquartile range, $0-8.0$ ]; $P=0.002$; Fig. 3). Representative images are shown in Figure 4. Reversible perfusion abnormalities were abolished in 7 of 45 MPI studies $(16 \%)$ after $\beta$-blockade. No significant relationship was found between defect reversibility and the hemodynamic response to adenosine before or after $\beta$-blockade ( $P \geq 0.06$ for all associations). Image quality was adequate to good, with no differences between the offand the on- $\beta$-blocker studies (quality score, $2.7 \pm 0.6$ vs. $2.8 \pm 0.4$, respectively; $P=0.6$ ). Body weight and body mass index did not significantly affect image quality $(P>$ 0.1 for both).

\section{Quantitative Analysis}

Quantitative assessment of regional myocardial perfusion showed a significant reduction in the median size of adenosine-induced defect from $32 \%(13 \%-54 \%)$ to $20 \%$ $(2 \%-41 \%)$ after $\beta$-blockade $(P<0.001$; Table 3$)$. The extent of reversible defect also decreased significantly after $\beta$-blockade (18\% [9\%-34\%] off $\beta$-blockers vs. $6 \%$ [0\%$19 \%$ ] on $\beta$-blockers; $P<0.001$ ).

No association was found between type and dose of $\beta$-blocker and perfusion changes after $\beta$-blockade $(P>$

\begin{tabular}{|c|c|c|c|}
\hline Parameter & Off $\beta$-blocker & On $\beta$-blocker & $P$ \\
\hline SSS & $12.0(7.0-17.0)$ & $9.0(6.0-17.5)$ & 0.03 \\
\hline SDS & $7.0(2.0-9.5)$ & $5.0(0-8.0)$ & 0.002 \\
\hline $\begin{array}{l}\text { Inducible defect } \\
\text { size (\%) }\end{array}$ & $32(13-54)$ & $20(2-41)$ & $<0.001$ \\
\hline $\begin{array}{l}\text { Reversibility } \\
\text { size (\%) }\end{array}$ & $18(9-34)$ & $6(0-19)$ & $<0.001$ \\
\hline
\end{tabular}

Data are presented as median, with interquartile ranges in parentheses. 


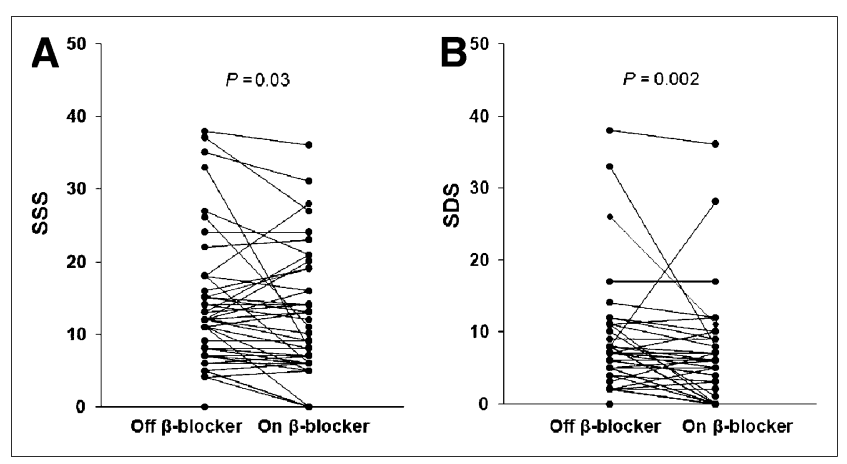

FIGURE 3. Individual SSSs (A) and SDSs (B) before and after $\beta$-blockade.

0.05 for all). There was no effect because of treatment period or order of randomization $(P=0.4$ and $P=0.2$, respectively).

\section{Detection of Angiographic Coronary Stenosis}

Results from $x$-ray coronary angiography were available in 36 of 45 patients. Obstructive CAD ( $\geq 50 \%$ stenosis in at least 1 major vessel) was identified in 32 patients, 12 of whom had single-vessel, fourteen 2-vessel, and six 3-vessel disease. Angiographically significant left main stem disease was identified in 1 patient. Reduction in defect reversibility (Table 4) was associated with a lower sensitivity for the detection of individual coronary stenosis after $\beta$-blockade (0.76 [95\% confidence interval, $0.65-0.88]$ vs. 0.58 [95\% confidence interval, 0.45-0.71], $P=0.03$; Fig. 5). A case example is shown in Figure 6.

\section{Side Effects}

Flushing (53\%), dyspnea (42\%), chest pain (36\%), and headache $(6 \%)$ were the most common side effects during adenosine stress off $\beta$-blockade. A significant reduction in the frequency and severity of adenosine-related side effects after $\beta$-blockade - as measured by the summed symptom score $(2.2 \pm 1.8$ vs. $1.5 \pm 1.2 ; P=0.003)$-was observed and was mainly due to a $31 \%$ reduction in the frequency of chest pain during the test. No adverse events were observed during or immediately after the adenosine infusion before or after $\beta$-blockade.

\section{ECG}

Complete ECG data were obtained in 43 of 45 patients. The frequency of new or worsening ischemic ECG changes during adenosine stress decreased significantly after $\beta$-blockade ( $9 / 43$ patients [21\%] vs. $1 / 43$ patients [2\%]; $P=0.008)$, and this decrease was paralleled by a reduction in reversibility score and defect size in 7 of 9 patients. All ECG abnormalities resolved spontaneously within $2 \mathrm{~min}$ after completion of the adenosine infusion.

\section{DISCUSSION}

This is the first study, to our knowledge, to demonstrate that $\beta$-adrenoceptor blockade is associated with a small reduction in the extent and severity of myocardial perfusion abnormality induced by intravenous adenosine in patients with obstructive CAD. As a result, $\beta$-blockade may reduce the sensitivity of adenosine MPI for the detection of coronary artery stenosis.

A few clinical studies have already provided evidence that $\beta$-blockade reduces the magnitude of perfusion abnormality on exercise and dobutamine MPI $(1,11)$. Therefore, discontinuation of $\beta$-blockers is recommended. The effect of $\beta$-blockade on primary coronary vasodilation is less clear, and recent observations are inconsistent $(4,7,8)$. In this study, $\beta$-blockade decreased cardiac workload both at rest and during adenosine stress. Moreover, $\beta$-blockers significantly reduced both the frequency and the magnitude of STsegment shift and the frequency and severity of chest pain during the adenosine infusion. These findings suggest that $\beta$-blockade decreases myocardial oxygen demand during vasodilator stress and may also reverse adenosine-induced ischemia in patients with obstructive CAD. More important,

FIGURE 4. $99 \mathrm{~m} T \mathrm{Tc}$-tetrofosmin adenosine-rest MPI (left) of 50-y-old man with history of previous percutaneous coronary intervention and recurrent symptoms. (A) Left ventricular tomograms show profound inducible perfusion abnormality in inferior wall (arrows). Tomograms also suggest transient left ventricular dilation, with quantitative stress-rest left ventricular volumes ratio of 1.51 . Inferior abnormality returns almost to normal at rest (C). (B) Inducible perfusion abnormality is no longer present after $\beta$-blockade. Stress and rest left ventricular volumes

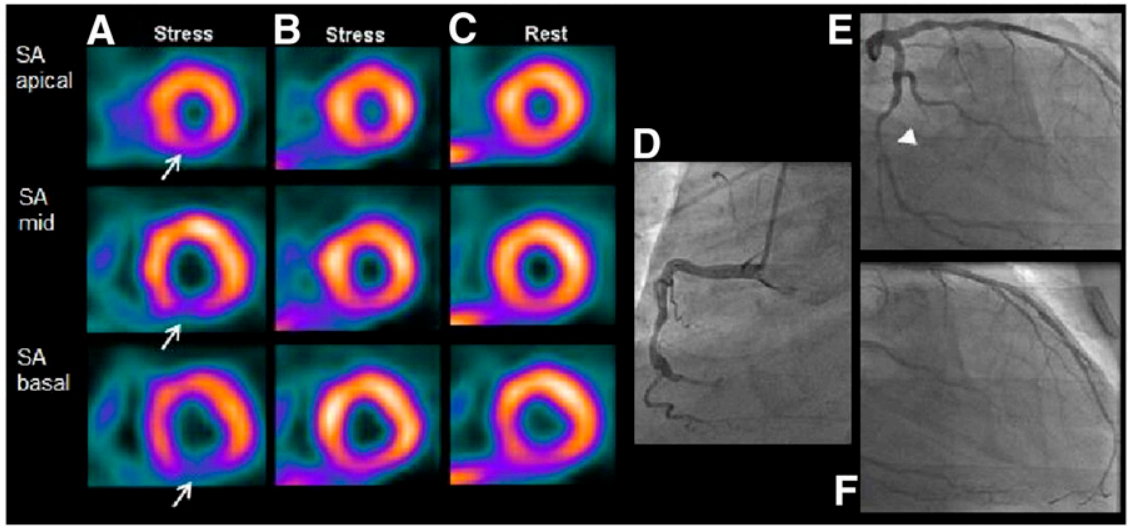
are also similar. X-ray coronary angiography reveals diseased right coronary artery (D) with occlusion in its posterolateral branch and severe distal left circumflex stenosis (E; arrowhead). No angiographically significant LAD disease (E-F) was observed. SA = short axis. 
TABLE 4. Defect Reversibility on Adenosine MPI Before and After $\beta$-Blockade in the Presence of Angiographically Significant Coronary Stenosis ( $n=32$ Patients)

\section{Off $\beta$-blocker}

On $\beta$-blocker Reversibility No reversibility Total $P$

$\begin{array}{llll}\text { Reversibility } & 21 & 0 & 21(66)\end{array}$

No reversibility $\quad 9 \quad 211$ (34)

$\begin{array}{lllll}\text { Total } & 30(94) & 2(6) & 32(100) & 0.01\end{array}$

Data in parentheses are percentages.

$\beta$-blockade interfered with the ability of adenosine to provoke myocardial blood flow heterogeneity resulting in attenuation of inducible perfusion abnormalities.

The observed reduction in the magnitude of reversibility by $\beta$-blockade was similar to that of a previous study of patients with CAD undergoing dipyridamole MPI after an intravenous dose of metoprolol (4). Both low and high doses of metoprolol significantly reduced the size and depth of perfusion defects and abolished reversibility in $29 \%$ of cases (4). Little is known about the effect of $\beta$-blockers on adenosine MPI. An early study has shown that antianginal medications including $\beta$-blockers reduce the sensitivity of adenosine MPI for detecting CAD (12). However, recent retrospective observations suggest that $\beta$-blockers have no effect on the extent, severity, or reversibility of adenosineinduced perfusion abnormalities (7). Although reversible defects were less extensive and severe in patients receiving $\beta$-blocker therapy, this difference was not statistically significant (7). Furthermore, these agents did not affect the frequency or severity of nonperfusion markers of ischemia. Possible reasons for these apparently discrepant results include differences in study design, the more pronounced $\beta$-blockade effect attained in our study, and differences in study populations.

An important finding of this study is that $\beta$-blockade significantly reduced the sensitivity of adenosine MPI for the detection of flow-limiting coronary stenosis. This observation is in agreement with a previous report demonstrating that $\beta$-blockade with metoprolol decreases the sensitivity of dipyridamole MPI for the detection of in-

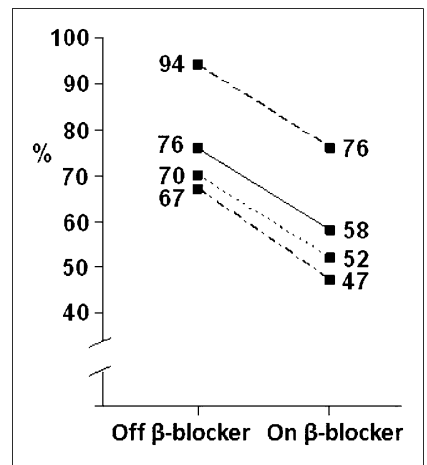

FIGURE 5. Sensitivity (\%) for detection of angiographic coronary stenosis ( $\geq 50 \%$ luminal diameter reduction) before and after $\beta$-blockade in left anterior descending (dotted line), left circumflex (dotted-dashed line), and right coronary (dashed line) arteries and in all coronary arteries combined (solid line). $P>0.05$ for individual vessel; $P=0.03$ for all vessels combined. dividual stenosis by $24 \%$ (4). Altogether, these results confirm earlier observations suggesting that antianginal drugs might adversely affect the diagnostic accuracy of vasodilator imaging $(5,6,12)$.

The precise mechanism by which $\beta_{1}$-adrenoceptor blockade may interfere with adenosine actions cannot be determined from this study. However, several mechanisms could explain the findings. First, $\beta$-blockers may attenuate adenosine-induced ischemia by increasing coronary collateral resistance and preventing blood from being shunted away from myocardium subtended by a stenotic coronary artery during maximal coronary vasodilation (13). Second, $\beta$-blockade may enhance subendocardial perfusion during adenosine hyperemia by prolonging diastole and reducing myocardial wall stress and thus extravascular compressive forces (14-16). And third, suppression of adrenergically mediated changes in vasomotor tone of coronary arteries may result in attenuation of perfusion heterogeneity during adenosine stress (17).

\section{Clinical Implications}

In the management of symptoms, documenting myocardial ischemia is an important step in the decision-making process and a prerequisite for elective coronary revascularization. In this setting, the assessment of myocardial perfusion on $\beta$-blocker therapy may be desirable, especially in patients with known CAD (18). However, it is unknown whether the prognostic value of adenosine MPI relates to baseline ischemia or to ischemia that is attenuated by $\beta$-blockade. As demonstrated in this study, $\beta$-blockers reduce the sensitivity of adenosine MPI for detecting individual coronary stenosis. This reduction suggests that the extent of coronary involvement may be underestimated in the initial evaluation of patients with suspected disease, and thus withholding $\beta$-blockers before adenosine MPI may be considered in these patients.

Discontinuation of $\beta$-blocker medication resulted in the withdrawal of 3 of 50 patients from the study because of exacerbation of anginal symptoms. Because discontinuation of $\beta$-blockers would be most relevant in the diagnostic setting, complications from $\beta$-blocker withdrawal might be less frequent (19-21). Nonetheless, abrupt cessation of these agents has been associated with an increased risk of angina and acute ischemic events, even in patients taking $\beta$-blockers for indications other than CAD (22). $\beta$-blockers should therefore be withdrawn before adenosine MPI, bearing in mind the risk of such complications.

\section{Study Limitations}

The aim of this study was to compare the results of adenosine MPI on and off $\beta$-blockers in patients with CAD and a high clinical suspicion of ischemia. Therefore, the findings of this study may not be transferable to a different population such as patients with a lower likelihood of disease undergoing diagnostic testing.

The study was conducted in patients treated with 3 different cardioselective $\beta$-blockers. It would have been 
FIGURE 6. Left ventricular tomograms and corresponding polar maps. (A) Extensive inducible perfusion abnormalities involving anterolateral region, inferior wall, and inferolateral region that improve at rest (C). (B) Abnormalities become less apparent after $25 \mathrm{mg}$ of atenolol, with almost complete normalization of perfusion in the anterior wall. $\mathrm{X}$-ray coronary angiography demonstrates $60 \%$ ostial stenosis of left anterior descending artery (D; circle), $50 \%$ stenosis of left circumflex artery and occluded OM1 (E; arrow), and proximal occlusion of right coronary artery $(F)$.

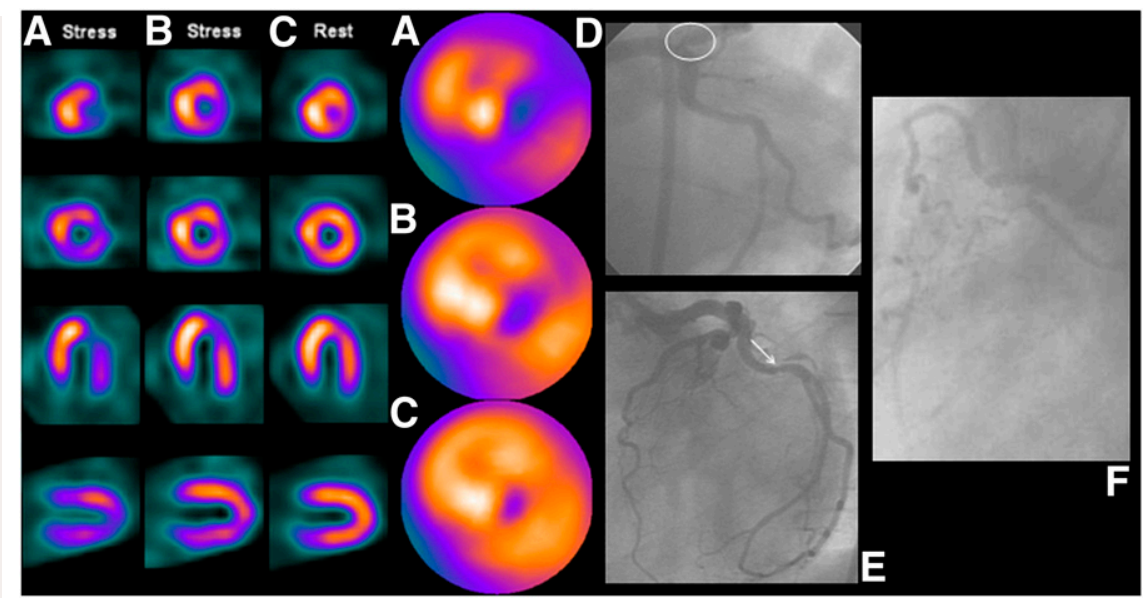

ideal to investigate each $\beta$-blocker separately but the study was too small for the data to be analyzed by individual drug.

The drug washout period of 5 half-lives may have been insufficient in some patients. Therefore, it is possible that there was some carryover effect and that a longer drug-free interval might have increased the differences between groups, although the main study conclusions would be unchanged.

To estimate the effect $\beta$-blockade might have on the detection of myocardial ischemia due to coronary stenosis, the results must be compared with a standard. X-ray coronary angiography is the best standard for coronary anatomy. The lack of quantitative assessment of the severity of coronary stenosis may be a weakness; however, clinical decision making is predominantly based on the visual assessment of the coronary angiogram.

\section{CONCLUSION}

Cardioselective $\beta$-blockers reduce the magnitude of adenosine-induced myocardial perfusion abnormalities in patients with CAD. Moreover, these agents reduce the sensitivity of adenosine MPI for the detection of flowlimiting coronary stenosis. These observations are of clinical relevance and support discontinuation of $\beta$-blockers before adenosine stress if knowledge of the unmodified hemodynamic consequence of underlying coronary disease is important to guiding further management.

\section{ACKNOWLEDGMENT}

This study was funded by a grant from the Clinical Research Committee, Royal Brompton and Harefield NHS Trust, London, United Kingdom.

\section{REFERENCES}

1. Muller-Suur R, Eriksson SV, Strandberg LE, Mesko L. Comparison of adenosine and exercise stress test for quantitative perfusion imaging in patients on $\beta$-blocker therapy. Cardiology. 2001;95:112-118.

2. Iskandrian AS, Heo J, Kong B, Lyons E. Effect of exercise level on the ability of thallium-201 tomographic imaging in detecting coronary artery disease: analysis of 461 patients. J Am Coll Cardiol. 1989;14:1477-1486.
3. Gupta NC, Esterbrooks DJ, Hilleman DE, Mohiuddin SM. Comparison of adenosine and exercise thallium-201 single-photon emission computed tomography (SPECT) myocardial perfusion imaging. The GE SPECT Multicenter Adenosine Study Group. J Am Coll Cardiol. 1992;19:248257.

4. Taillefer R, Ahlberg AW, Masood Y, et al. Acute $\beta$-blockade reduces the extent and severity of myocardial perfusion defects with dipyridamole Tc-99m sestamibi SPECT imaging. J Am Coll Cardiol. 2003;42:14751483.

5. Sharir T, Rabinowitz B, Livschitz S, et al. Underestimation of extent and severity of coronary artery disease by dipyridamole stress thallium-201 single-photon emission computed tomographic myocardial perfusion imaging in patients taking antianginal drugs. J Am Coll Cardiol. 1998;31:15401546

6. Bottcher M, Refsgaard J, Madsen MM, et al. Effect of antianginal medication on resting myocardial perfusion and pharmacologically induced hyperemia. $\mathrm{J} \mathrm{Nucl}$ Cardiol. 2003;10:345-352.

7. Lakkireddy D, Aronow WS, Bateman T, McGhee I, Nair C, Khan IA. Does $\beta$ blocker therapy affect the diagnostic accuracy of adenosine singlephoton-emission computed tomographic myocardial perfusion imaging? Am J Ther. 2008;15:19-23.

8. Ozdemir M, Yazici GE, Turkoglu S, Timurkaynak T, Cengel A. Metoprolol does not effect myocardial fractional flow reserve in patients with intermediate coronary stenoses. Int Heart J. 2007;48:477-483.

9. Campeau L. Letter: grading of angina pectoris. Circulation. 1976;54:522523.

10. Cerqueira MD, Weissman NJ, Dilsizian V, et al.; American Heart Association Writing Group on Myocardial Segmentation and Registration for Cardiac Imaging. Standardized myocardial segmentation and nomenclature for tomographic imaging of the heart: a statement for healthcare professionals from the Cardiac Imaging Committee of the Council on Clinical Cardiology of the American Heart Association. Circulation. 2002; 105:539-542.

11. Geleijnse ML, Elhendy A, Fioretti PM, Roelandt JR. Dobutamine stress myocardial perfusion imaging. J Am Coll Cardiol. 2000;36:2017-2027.

12. Sundereswaran L, He ZX, Verani MS, Mahmarian JJ. Influence of antianginal medications on the detection of coronary artery disease as assessed by singlephoton emission computed tomography combined with adenosine stress [abstract]. Circulation. 1996;94(suppl I):303-304.

13. Billinger M, Fleisch M, Eberli FR, Meier B, Seiler C. Collateral and collateral-adjacent hyperemic vascular resistance changes and the ipsilateral coronary flow reserve: documentation of a mechanism causing coronary steal in patients with coronary artery disease. Cardiovasc Res. 2001;49:600608.

14. Koepfli P, Wyss CA, Namdar M, et al. $\beta$-adrenergic blockade and myocardial perfusion in coronary artery disease: differential effects in stenotic versus remote myocardial segments. J Nucl Med. 2004;45:1626-1631.

15. Billinger M, Seiler C, Fleisch M, Eberli FR, Meier B, Hess OM. Do $\beta$-adrenergic blocking agents increase coronary flow reserve? J Am Coll Cardiol. 2001;38: 1866-1871.

16. Bottcher M, Czernin J, Sun K, Phelps ME, Schelbert HR. Effect of $\beta_{1}$ adrenergic receptor blockade on myocardial blood flow and vasodilatory capacity. $\mathrm{J} \mathrm{Nucl}$ Med. 1997;38:442-446. 
17. Di Carli MF, Tobes MC, Mangner T, et al. Effects of cardiac sympathetic innervation on coronary blood flow. N Engl J Med. 1997;336:1208-1215.

18. Madjlessi-Simon T, Mary-Krause M, Fillette F, Lechat P, Jaillon P. Persistent transient myocardial ischemia despite $\beta$-adrenergic blockade predicts a higher risk of adverse cardiac events in patients with coronary artery disease. J Am Coll Cardiol. 1996;27:1586-1591.

19. Walker PR, Marshall AJ, Farr S, Bauminger B, Walters G, Barritt DW. Abrupt withdrawal of atenolol in patients with severe angina: comparison with the effects of treatment. Br Heart J. 1985;53:276-282.
20. Eisele G, Gilmore LL, Blanchard EB. Close clinical observation minimizes the complications of $\beta$-blocker withdrawal. Ann Pharmacother. 1994;28:849-851.

21. Croft $\mathrm{CH}$, Rude RE, Gustafson N, et al. Abrupt withdrawal of $\beta$-blockade therapy in patients with myocardial infarction: effects on infarct size, left ventricular function, and hospital course. Circulation. 1986;73: 1281-1290.

22. Psaty BM, Koepsell TD, Wagner EH, LoGerfo JP, Inui TS. The relative risk of incident coronary heart disease associated with recently stopping the use of $\beta$-blockers. JAMA. 1990;263:1653-1657. 